\title{
In vitro assessment of six aspiration catheters using a distal protection filter
}

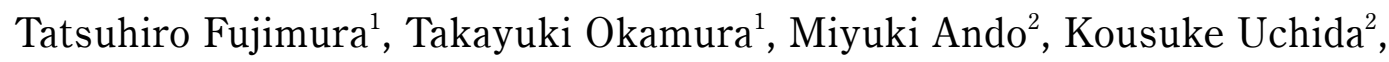 \\ Takashi Tone ${ }^{2}$, Fumio Yonezawa ${ }^{2}$, Masafumi Yano ${ }^{1}$ \\ ${ }^{1}$ Division of Cardiology, Department of Medicine and Clinical Science, \\ Yamaguchi University Graduate School of Medicine, Yamaguchi, Japan \\ ${ }^{2}$ Division of Cardiology, Hagi Civil Hospital, Yamaguchi, Japan
}

\begin{abstract}
Background: We assessed performance of 6 aspiration catheters for distal embolization using a distal protection filter in an in vitro experiment. In acute myocardial infarction, a distal protection filter is used for lesions likely to induce a distal embolism. Which aspiration cathether is most effective when used with a distal protection filter remains still unclear.
\end{abstract}

Methods: A 0.5- $\mathrm{cm}^{3}$ bolus of gelatin as a model of stagnant pools of coronary plaque debris was captured in the distal protection filter and aspirated by 6 aspiration catheters. We measured and compared the length of the suspended embolus matter.

Results: Among the 6 catheters evaluated, the use of the Export Advance catheter (Medtronic) resulted in significantly shorter lengths of the suspended embolus matter compared to the use of the TVAC II (Nipro), Thrombuster III SL (Kaneka), and Rebirth Pro (Goodman) catheters $(p<0.01)$. The residual embolus matter in all cases had drained distally to the distal protection filter when the filter was retrieved.

Conclusions: The use of the Export Advance catheter showed better performance using a distal protection filter in this in vitro experiment, and its use might be more effective in preventing distal embolisms in combination with a distal protection filter. (Cardiol J 2016; 23, 6: 667-672)

Key words: percutaneous coronary intervention, distal embolization, acute myocardial infarction, aspiration catheters, distal protection device and filter no-reflow

\section{Introduction}

The no-reflow phenomenon indicates a periprocedural myocardial infarction (MI) even with the successful dilatation of occluded arteries, resulting in a poor prognosis $[1,2]$. The reported frequency of the no-reflow phenomenon was $13 \%$ in patients with an ST-segment elevation MI (STEMI) who had under- gone a primary percutaneous coronary intervention (PCI) $[2,3]$. The main cause of the no-reflow phenomenon is a distal embolism due to thrombi and/or debris from a ruptured plaque [4]. A distal embolism during a primary PCI results in a reduction of myocardial perfusion and poor clinical outcomes [5-7]. Various distal protection filters and aspiration catheters were developed to prevent distal embolisms.

Address for correspondence: Tatsuhiro Fujimura, MD, Division of Cardiology, Department of Medicine and Clinical Science, Yamaguchi University Graduate School of Medicine. 1-1-1 Minami-Kogushi, Ube, Yamaguchi 755-8505, Japan, tel: +81-836-22-2248, fax: +81-0836-22-2246, e-mail: tafujimura-circ@umin.ac.jp

Received: 24.06 .2016

Accepted: 16.09.2016 
However, the clinical benefits of the distal protection filters and aspiration catheters during primary PCI in patients with STEMI have been a matter of discussion. The phenomenon of filter no-reflow has been reported as one of the causes of failure in accomplishing successful Thrombolysis in Myocardial Infarction (TIMI)-3 flow when using these embolism-prevention devices [8,9].

We speculated that the use of an aspiration catheter device and a distal protection filter device may not be enough to retrieve embolic debris and aspirate the suspended embolus matter in the proximal portion of the distal protection filter device when filter no-reflow is observed. Although several types of aspiration catheters have been developed, it is not clear how much embolus matter any of the aspiration catheters can aspirate with the use of the distal protection filter.

In the present study, we investigated the efficacy of six aspiration catheters for retrieving suspended embolus matter in a proximal portion of a distal protection filter and how much of the embolus matter is left behind after aspiration.

\section{Methods}

The experiment was performed using a simulated vascular system (Wet Track Glass Model, Medtronic, Minneapolis, MN). The system, made of glass and a polyvinyl chloride tube (4 $\mathrm{mm}$ dia.), produces a steady flow that mimics the human cardiovascular system. The flow velocity was $3.38 \mathrm{~m} / \mathrm{s}$ (Fig. 1) [10].

A Filtrap distal protection filter $(5.0 \mathrm{~mm}$ dia., Nipro, Osaka, Japan) was used. The device has a 35-mm-long distal radiopaque floppy guidewire with a $1.15-\mathrm{mm}$ dia. distal tip fixed in a spindle-shaped spiral wire basket with a porous polyurethane filter membrane (Fig. 1). The filter was delivered to the middle of the simulated vascular tube. As a model of stagnant pools of coronary plaque debris, $0.5 \mathrm{~cm}^{3}$ of brown gelatin was injected into the tube by the aspiration catheter. This amount of gelatin was sufficient to obstruct the simulated vascular flow through the tube. The brown gelatin was soft enough to change the shape. The embolus matter was captured in Filtrap by the artificial flow and was aspirated by each aspiration catheter device through the original syringe packaged with the aspiration catheter. The length of the suspended embolus matter was defined as the proximal edge of the brown gelatin from the Filtrap's distal end and was measured for each sample (Fig. 2).

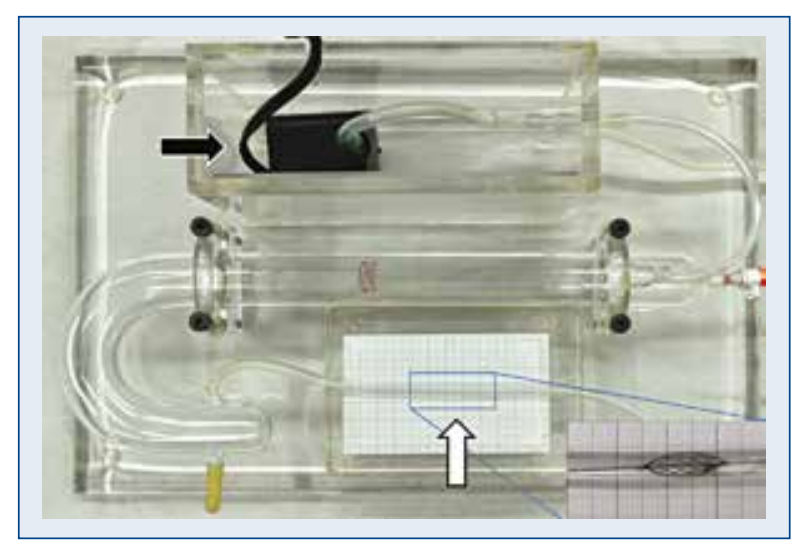

Figure 1. Simulated vascular system (Wet Track Glass Model, Medtronic). The system is made of glass and a polyvinyl chloride tube and produced a continuous flow that mimics the human cardiovascular system. The motor that creates the steady current is indicated by the black arrow. The location at which Filtrap is deployed in the simulated vascular tube is shown by the white arrow.

Six aspiration catheters were evaluated: Export Advance (Medtronic), Thrombuster III SL/GR (Kaneka Medix, Osaka, Japan), Eliminate 3 (Terumo, Tokyo), TVAC II (Nipro, Osaka, Japan), and Rebirth Pro (Goodman, Aichi, Japan). The length of the tip, the length from the tip to the distal end of the aspiration port, and the aspiration lumen area were originally measured by digital venire caliper for each catheter (Fig. 3).

The experiment was performed 9 times for each catheter, with 3 interventional cardiologists (A, B and C) each executing 3 procedures per aspiration device. The length of each of the suspended emboli was measured independently by 2 observers. The value reported for each catheter is the average of the measurements from 2 observers.

\section{Statistical analysis}

Comparisons between aspiration catheters were made using the Kruskal-Wallis test. P-values $<0.05$ were considered significant. All numerical values are expressed as the mean \pm standard deviation. An intercorrelation coefficient was used to determine the inter-observer agreement of 2 observers. Spearman's rank-correlation coefficient was used to test the agreement between the length from the tip to the distal end of the aspiration port and the length of the suspended embolus matter. Statistical analyses were performed using SPSS (Statistical Package for Social Science) software for Windows version 16.0 (SPSS, Chicago, IL). 


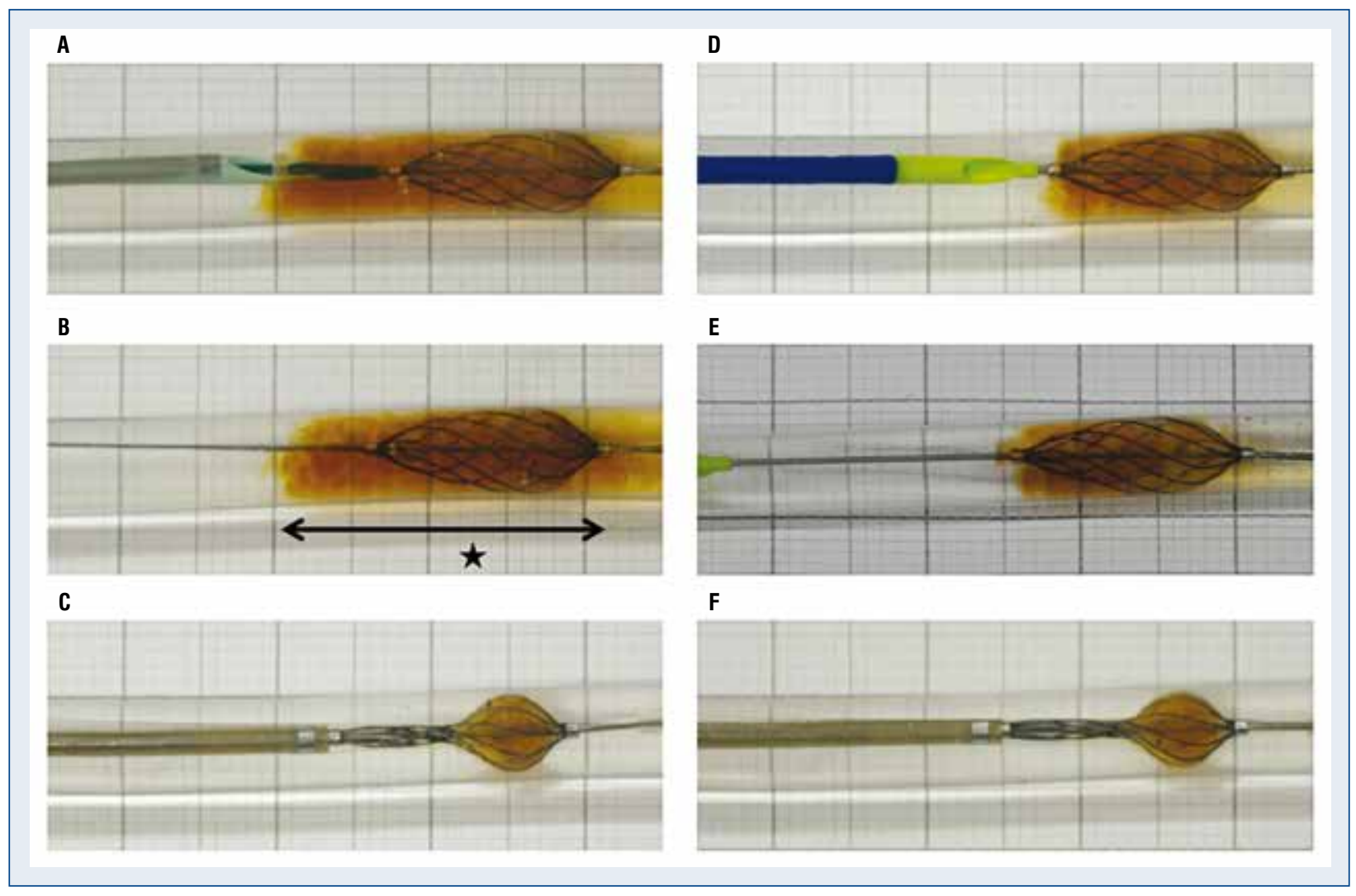

Figure 2. Representative examples. Images immediately after aspiration by the Thrombuster III SL catheter and Export Advance catheter, respectively (A, D). The suspended embolic matter for the Thrombuster III SL catheter and Export Advance catheter, respectively (B, E). Final images of the retrieval of Filtrap with an expropriation catheter (C, F). Panels $\mathbf{C}$ and $\mathbf{F}$ are representative for all samples tested, as retrieving the distal protection device produced similar results. The length of the suspended embolus matter is indicated by the black star.

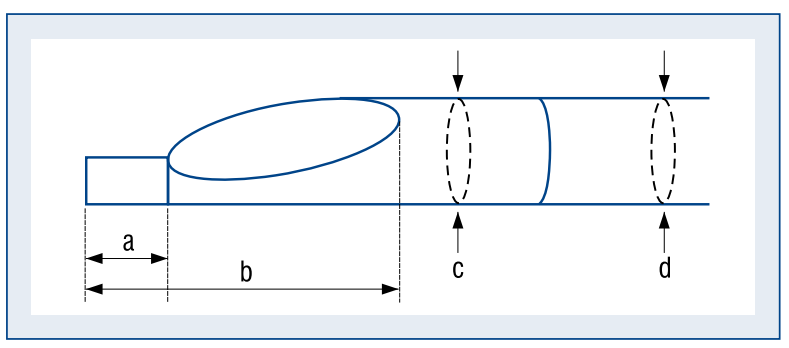

Figure 3. Schema of the aspiration device and measurement. The lengths of " $a$ " and " $b$ " indicate the tip length and the length from the tip to the distal end of the aspiration port, respectively. The areas of " $c$ " and " $d$ " indicate the aspiration lumen area of the distal and the proximal ends, respectively.

\section{Results}

The results are presented in Table 1 . The Export Advance catheter has the shortest length of the tip and shortest distance from the tip to the dis- tal end of the aspiration port compared to the other five catheters. The Thrombuster III GR catheter has the largest aspiration lumen area compared to the other catheters. The lengths of the suspended embolus matter for each aspiration catheter were: Export Advance, $15.4 \pm 1.0 \mathrm{~mm}$; Thrombuster III GR, $18.1 \pm 1.2 \mathrm{~mm}$; Eliminate 3, $18.4 \pm 1.2 \mathrm{~mm}$; TVAC II, $19.1 \pm 1.5$; Thrombuster III SL, $19.4 \pm$ $\pm 0.9 \mathrm{~mm}$; and Rebirth Pro, $21.1 \pm 2.2 \mathrm{~mm}$.

Among the 6 aspiration catheters evaluated, the use of the Export Advance catheter tended to provide a shorter length of suspended embolus matter than the other catheters. The use of the Export Advance resulted in significantly shorter lengths of suspended embolus matter compared to the use of the TVAC II catheter, the Thrombuster III SL catheter and the Rebirth Pro catheter (p < 0.01) (Fig. 4).

There were no significant differences in the measurements of the length of the suspended embolus matter in each aspiration catheter among 


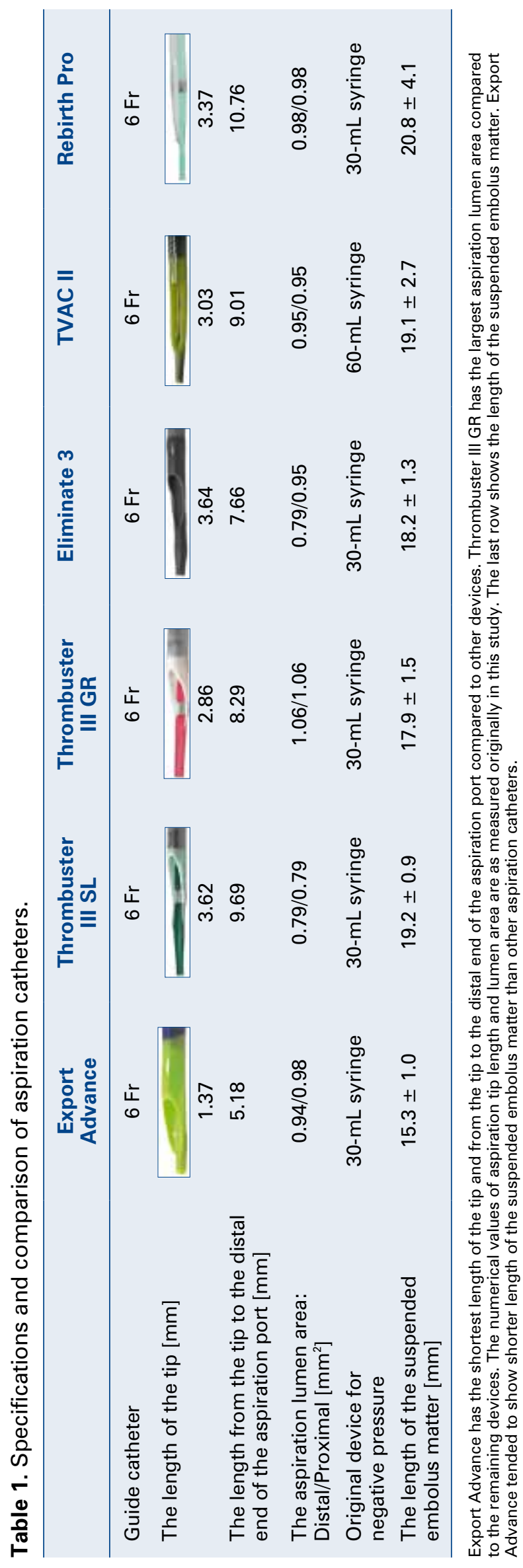

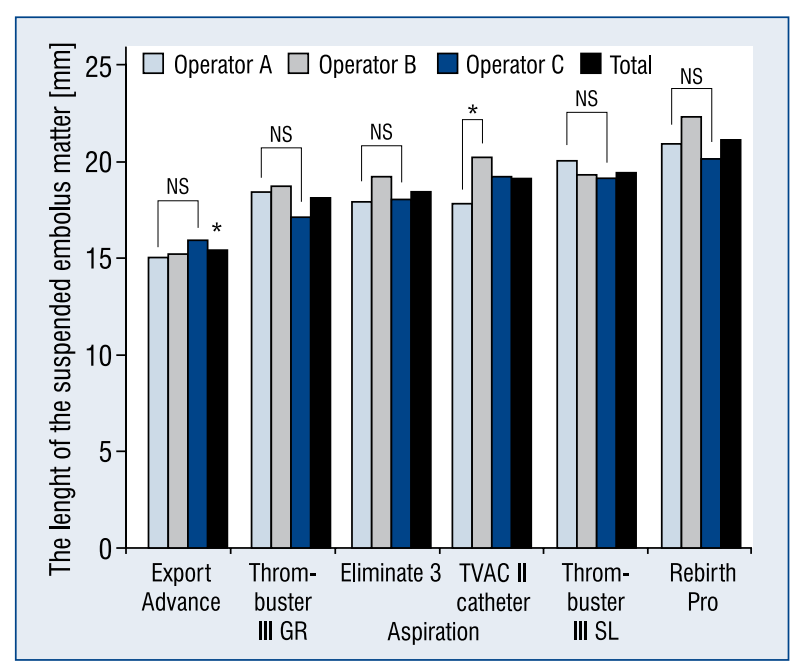

Figure 4. The length of the suspended embolus matter in the distal protection filter after the use of each aspiration catheter; ${ }^{*} p<0.01$ compared to the use of the TVAC II, the Thrombuster III SL and the Rebirth Pro catheters; star - significantly different between Operators $\mathrm{A}$ and $\mathrm{B}$.

the 3 interventional cardiologists except TVAC II, which showed a significant difference in the procedures obtained by operators A and B (Fig. 4). The inter-observer agreement of 2 Observers for the measurement of the length of the suspended embolic matter was 0.995 .

The suspended embolus matter in all cases had drained distally to Filtrap when the distal protection device was retrieved (Supplementary Video - see journal website).

Spearman's rank-correlation coefficient also indicated a significant correlation between the length from the tip to the distal end of the aspiration port and the length of suspended embolus matter (Fig. 5).

\section{Discussion}

The main finding of the study is that the use of the Export Advance catheter tended to provide a shorter length of the suspended embolus matter than other aspiration catheters. The same ability for aspiration was shown by the Export Advance catheter regardless of the operator's experience. This is likely because the Export Advance catheter has the shortest distal tip among the 6 catheters tested. The shorter distal tip of aspiration catheter can aspirate suspended embolus matter most nearby distal protection device. 


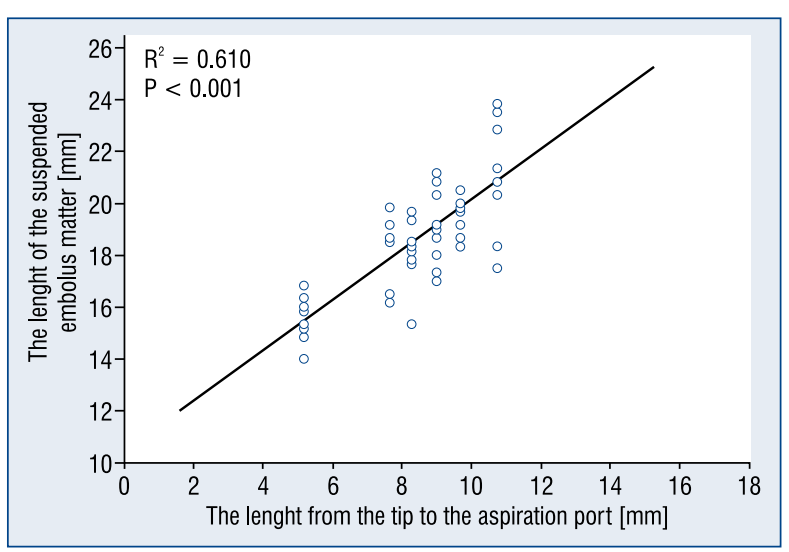

Figure 5. Spearman's rank-correlation coefficient indicated a significant correlation between the length from the tip to the distal end of the aspiration port and the length of suspended embolus matter $(p<0.001)$.

Our results also showed that the residual embolus matter, which could not be aspirated by any of the aspiration catheters, drained to the distal site of the Filtrap distal protection filter. This result may reflect one of the mechanisms potentially underlying the inability to prevent distal embolization in STEMI with a distal protection filter and an aspiration device.

In clinical practice, despite the use of a distal protection device and an aspiration device, same cases showed severe final TIMI flow grade 0 or 1 for STEMI [11]. Several recent clinical trials demonstrated that the routine therapy of intracoronary aspiration thrombectomy and distal protection during primary PCI for acute MI has not improved clinical outcomes despite the removal of debris, except in cases with a high risk of distal embolization $[11,12]$. At present, the distal protection device is not recommended to be used as routine adjunctive therapy in primary PCI procedures with stenting in patients with a STEMI in the native circulation, or in the routine performance of thrombus aspiration in patients undergoing a primary PCI.

Recent data suggest that a primary PCI with thrombus aspiration in MI patients was more likely to result in a higher TIMI grade in the infarctrelated artery and a lower in-hospital mortality rate compared to primary PCI without aspiration [13]. Moreover, another report demonstrated that distal protection during PCI effectively retrieved the embolic debris and preserved the index of microcirculatory resistance in patients with an acute anterior STEMI [14]. Embolic protection devices are designed to be able to retrieve atherothrom- botic debris and prevent its distal embolisation, but their effect on clinical outcomes has not been established, except for saphenous vein bypass grafts $[6,7,15-17]$.

Due to the fact that we encounter cases that result in coronary no-reflow among patients with eccentric plaques, positive remodeling and lipidrich plaque with attenuation regardless of whether STEMI is present, we consider it reasonable to conduct an aspiration thrombectomy in patients with a large thrombus burden, and the use of a distal protection device in patients with ruptured plaque likely leads to distal embolism [3, 6, 22]. Although no-reflow can occur not only due to a distal embolism from a thrombus and/or ruptured plaque but also endothelial damage, tissue edema, tissue contracture, oxygen free radicals and intravascular plugging by fibrin, platelets or leukocytes [4], the appropriate use of aspiration catheters and distal protection devices might prevent at least coronary no-flow and achieve better coronary flow during a primary PCI. Clinicians should be aware of the varying performance of different aspiration devices and distal protection devices. The results of the present study demonstrated that the Export Advance aspiration catheter might be more effective than other aspiration catheters at present when filter no-reflow is observed.

The results of our present in vitro experiment demonstrated that as the length from the tip to the distal end of the catheter's aspiration port shortens, the aspiration catheter can be used closer to the distal protection device and thus aspirate more of the suspended embolus matter captured in the distal protection device. When filter no-reflow occurs, an improved aspiration catheter that has a shorter tip length is needed.

In addition, the results of our study indicate that it is not possible to completely retrieve the debris and prevent distal embolization by using the current distal protection device. One of the reasons for this is that the residual embolus matter, which could not be aspirated by any of the aspiration catheters, drained to the distal site of the distal protection filter device. Our findings indicate that when the filter no-reflow phenomenon occurs, a shorter-tip aspiration catheter should be used to aspirate the debris to the very distal end of the filter device, to maximize the effect of the distal protection filter. In the future, the development of improved distal protection devices and aspiration devices with shorter tips may make it possible to completely aspirate a suspended embolus when filter no-reflow is present. 


\section{Limitations of the study}

The present study has several limitations. First, although the Filtrap embolic protection filter is frequently used in Japan (accounting for $92 \%$ of the distal protection devices in the field of coronary intervention in Japan), it may not be widely used elsewhere. In addition, we did not determine the relationships between other distal protection devices and the aspiration catheters. We tested the efficacy of the aspiration catheters using Filtrap only. However, we consider that the basic structure of Filtrap is essentially the same as of other commercially available embolic protection filters such as FilterWire (EPI, Boston Scientific, Natick, MA), Angioguard (Cordis, Miami Lakes, FL), and Parachute (Tri-Med, Osaka, Japan).

Second, the embolus matter in this in vitro study was artificial gelatin. There may be significant differences between such an experimental model and diseased human coronary arteries. It is necessary to test each aspiration catheter in multicenter clinical trials.

\section{Conclusions}

The use of the Export Advance aspiration catheter with the shortest length from the tip to the distal end of the aspiration port showed better performance with the distal protection filter in this in vitro experiment, and its use might be more effective to prevent distal embolisms in combination with a distal protection filter.

\section{Acknowledgements}

The circuit simulator was supplied by Medtronic, Inc. We thank Mr. Yasuhiro Kajiwara, Mr. Takeshi Tomiyoshi, and Mr. Atsushi Shiraishi for the preparation of and assistance with the in vitro experiments.

\section{Conflict of interest: None declared}

\section{References}

1. Kloner RA, Gantone CE, Jennings RB. The "no-reflow" phenomenon after temporary coronary occlusion in the dog. J Clin Invest, 1974; 54: 1496-1508.

2. Choo EH, Kim PJ, Chang $\mathrm{K}$ et al. The impact of no-reflow phenomena after primary percutaneous coronary intervention: A time-dependent analysis of mortality. Coron Artery Dis, 2014; 25: 392-398.
3. Tanaka A, Kawarabayashi T, Nishibori Y et al. No-reflow phenomenon and lesion morphology in patients with acute myocardial infarction. Circulation, 2002; 105: 2148-2152.

4. Shereif H. Rezkalla, Robert A. Kloner. No-reflow phenomenon. Circulation, 2002; 105: 656-662.

5. Muramatsu T, Kozuma K, Tsukahara R et al. Comparison of myocardial perfusion by distal protection before and after primary stenting for acute myocardial infarction: Angiographic and clinical results of a randomized controlled trial. Catheter Cardiovasc Interv, 2007; 70: 677-682.

6. Mizote I, Ueda Y, Ohtani T, Shimizu M et al. Distal protection improved reperfusion and reduced left ventricular dysfunction in patients with acute myocardial infarction who had angioscopically defined ruptured plaque. Circulation, 2005; 112: 1001-1007.

7. Grube E, Gerckens U, Yeung AC et al. Prevention of distal embolization during coronary angioplasty in saphenous vein grafts and native vessels using porous filter protection. Circulation, 2001; 104: 2436-2441.

8. Porto I, Choudhury RP, Pillay P et al. Filter no reflow during percutaneous coronary interventions using the Filterwire distal protection device. Int J Cardiol, 2006; 109: 53-88.

9. Porto I, Belloni F, Niccoli G et al. Filter no-reflow during percutaneous coronary intervention of saphenous vein grafts: Incidence, predictors and effect of the type of protection device. Euro Intervention, 2011; 7: 955-961.

10. Isshiki T, Kozuma K, Kyono $\mathrm{H}$ et al. Initial clinical experience with distal embolic protection using "Filtrap," a novel filter device with a self-expandable spiral basket in patients undergoing percutaneous coronary intervention. Cardiovasc Intervent Ther, 2010; 26: 12-17.

11. Kelbak H, Terkelsen CJ, Helqvist S et al. Randomized comparison of distal protection versus conventional treatment in primary percutaneous coronary intervention: The drug elution and distal protection in ST-elevation myocardial infarction (DEDICATION) trial. J Am Coll Cardiol, 2008; 51: 899-905.

12. Fröbert O, Lagerqvist B, Olivecrona GK et al. Thrombus aspiration during ST-segment elevation myocardial infarction. N Engl J Med, 2013; 369: 1587-1597.

13. Shiraishi J, Kohno Y, Nakamura T et al. Clinical impact of thrombus aspiration during primary percutaneous coronary intervention in acute myocardial infarction with occluded culprit. Cardiovasc Interv Ther, 2015; 30: 22-28.

14. Ito N, Nanto S, Doi Y, Kurozumi Y et al. Distal protection during primary coronary intervention can preserve the index of microcirculatory resistance in patients with acute anterior ST-segment elevation myocardial infarction. Circ J, 2011; 75: 94-98.

15. Stone GW, Webb J, Cox DA et al. Distal microcirculatory protection during percutaneous coronary intervention in acute ST-segment elevation myocardial infarction: A randomized controlled trial. JAMA, 2005; 293: 1063-1072.

16. Limbruno U, Micheli A, De Carlo M et al. Mechanical prevention of distal embolization during primary angioplasty: Safety, feasibility, and impact on myocardial reperfusion. Circulation, 2003; 108: 171-176.

17. Nakamura T, Kubo N, Seki Y et al. Effects of distal protection device during primary stenting in patients with acute anterior myocardial infarction. Circ J, 2004; 68: 763-768. 